\title{
Some new discrete biorthogonal wavelets constructed with Laguerre polynomials
}

\author{
Atemangoh Bruno Peachap ${ }^{1,2} \cdot$ Daniel Tchiotsop $^{2} \cdot$ Valérie Louis-Dorr $^{3} \cdot$ Didier Wolf $^{3}$
}

Received: 12 March 2020 / Accepted: 24 July 2020 / Published online: 3 August 2020

(c) Springer Nature Switzerland AG 2020

\begin{abstract}
In this study, we present a new family of discrete wavelets which are constructed with the help of Laguerre polynomials and the Daubechies biorthogonal wavelets construction method. Our aim is to propose the discrete version of some previously constructed continuous Laguerre wavelets and also to present a method of discrete wavelets construction by several iterations. With this scheme, we use two different sets of finite impulse response filters for the signal decomposition and their duals for reconstruction. The quadruplet finite impulse response filters respect the anti-aliasing and the perfect reconstruction conditions, and at the same time, they resemble as much as possible the continuous Laguerre wavelets when using the cascade algorithm. We use the mean squared error, the maximum deviation, and the standard deviation to quantify the similarity between the continuous Laguerre wavelets and the constructed discrete Laguerre wavelets. The results show that, they are both the same wavelets due to the small nature of these parameters. Our method is important because, it can permit the determination of the finite impulse response filter coefficients corresponding to many other continuous wavelets.
\end{abstract}

Keywords Laguerre wavelets · Biorthogonal wavelets · Cascade algorithm · FIR filters

\section{Introduction}

Wavelets are a very vital tool in the field of signal processing and they are applied in several domains like medicine and engineering to perform tasks such as data compression, signal denoising, signal feature extraction for classification, etc. In recent years, many wavelets have been constructed by different researchers for various purposes since they have proven to be a better signal analysis tool than the Fourier transform. More so, they are popular due to the existence of algorithms that can compute wavelet coefficients fast such as the fast wavelet transform (FWT) algorithm [1, 2]. Fundamentally, we have the continuous wavelets (small waves of zero mean, that oscillate and varnish) and the discrete wavelets (finite impulse response filters). The cascade algorithm is a technique whereby the continuous wavelets can be obtained from the FIR filters through several iterations. We are proposing a method whereby, the FIR filter coefficients of the discrete wavelets can be obtained from its continuous wavelets.

It was long suggested that Laguerre functions could be used to construct wavelets [3].

Recently, some new continuous wavelets were constructed with Laguerre functions and applied in the classification of electroencephalogram (EEG) signals $[4,5]$. The applications of the continuous wavelets are however limited because of the limitations of the continuous wavelet transform (CWT) algorithm (it is slower, redundant and requires more computational space and time) compared to the FWT algorithm. If we want to implement the constructed

$\triangle$ Atemangoh Bruno Peachap, peachapbruno2013@gmail.com | 'Unité de Recherche de Matière Condensée D'Electronique Et de Traitement du Signal (UR-MACETS), Faculty of Science, University of Dschang, P.O. Box 69, Dschang, Cameroon. ${ }^{2}$ Unité de Recherche D'Automatique Et D'Informatique Appliquée (LAIA), IUT-FV de Bandjoun, University of Dschang, P.O. Box 134, Bandjoun, Cameroon. ${ }^{3}$ Centre de Recherche en Automatique de Nancy (CRAN), UMR CNRS 7039, ENSEM, Université de Lorraine, Nancy, France. 
wavelets using the FWT algorithm, their corresponding finite impulse response filter (FIR) coefficients have to be determined because discrete wavelets correspond to the coefficients of FIR filters [6]. It is in this perspective that we study how we can determine the FIR filter coefficients corresponding to the constructed continuous Laguerre wavelets. Once this is done, the wavelets can be implemented with the FWT algorithm in signal analysis tasks like filtering, denoising, compression, etc.

The question is "how do we determine the FIR filter coefficients of continuous wavelets?" We know that in an exact reconstruction sub-band coding Scheme with orthonormal bases of compactly supported wavelets, the analysis and synthesis filters are the same. Meanwhile, with biorthogonal wavelets, the synthesis and analysis filter coefficients are not the same [7]. This permits flexibility during the construction of compactly supported wavelets with some desired attributes like the modification of the number of vanishing moments or its shape [8]. Many authors have devised several techniques to construct wavelets in the literature, starting from the simplest Haar wavelet [9], through the Morlet wavelet for CWT to Stephan Mallat and Ingrid Daubechies wavelets for DWT [10]. In recent years, some authors have designed many other wavelets [11]. Most of these wavelets are designed to suit certain conditions or constraints like the number of filter tabs, varnishing moments and regularity. They also serve diverse purposes and applications $[12,13]$.

This work is a continuation of $[4,5]$ where the authors constructed some new continuous wavelets with the Laguerre functions and applied them in the classification of EEG signals with good classification accuracy results. In [4], we constructed continuous wavelets with Laguerre polynomials which respect the admissibility and regularity conditions of wavelets. In [5], the continuous Laguerre wavelets are applied in the detection of epilepsy in electroencephalographic (EEG) signals with the help of artificial neural networks and support vector machines with good classification accuracy results. In this work, we have determined the finite impulse response (FIR) filter coefficients that correspond to discrete Laguerre wavelets and they can be useful in several signal processing applications. This paper is divided as follows. In part one, we Introduce this paper, part two is a presentation of the materials and methods used in this work. In part three, we present the results obtained from this research and we discuss them. We end this paper with a conclusion.

\section{Materials and methods}

\subsection{The Wavelet construction method.}

Laguerre wavelets are part of the family of the Hermitian hat wavelet, which are defined by generalized Laguerre functions. They are not compactly supported, but their effective support is $[-5,5]$ and can be rendered compactly supported if it is defined only within its effective interval. They are defined as follows [4]:

For even values,

$\psi_{2 n}(x)=J_{n} e^{-\frac{(x-5)^{2}}{2}} L_{n}^{\left(-\frac{1}{2}\right)}\left((x-5)^{2}\right)$

$\forall x \in\left[\begin{array}{ll}0 & 10\end{array}\right]$

$\psi_{2 n}(x)=0$ otherwise

For odd values,

$\psi_{2 n+1}(x)=K_{n}(x-5) e^{-\frac{(x-5)^{2}}{2}} L_{n}^{\left(\frac{1}{2}\right)}\left((x-5)^{2}\right)$

$\forall x \in\left[\begin{array}{ll}0 & 10\end{array}\right]$

$\psi_{2 n}(x)=0$ otherwise

where $J_{n}$ and $K_{n}$ are normalization constants such that the $L^{2}$ norm of $\psi$ is 1 [5].

$J_{n}=\left(n ! \sqrt{\pi} 2^{n}\right)^{-\frac{1}{2}}\left(\frac{43}{\pi} n^{2}-\frac{98 \pi}{9} n+15^{\frac{3}{4}} \pi\right)$

$K_{n}=\left(n ! \sqrt{\pi} 2^{n}\right)^{-\frac{1}{2}}\left(\frac{53 \pi}{130} n^{2}-\frac{7}{\pi} n+\frac{19}{2 \pi}\right)$

$L_{n}$ is the generalized Laguerre polynomial $[14,15]$. It is worth noting that these wavelets have a similar shape to the Gaussian wavelets though they are different in their expressions [16]. The absolute mean square error difference between them is also greater than zero.

The next step is to get a scaling function $\phi(x)$, which is a function that can verify the refinement equation below.

$\phi(x)=\sum_{n \in \mathbb{Z}} C_{n} \phi(2 x-n)$

The scaling function coefficients $C_{n}$ in (5) cannot be any arbitrary sequence. Orthogonality of the $\phi_{0, k}$ immediately implies

$\sum_{n} C_{n} C_{n+2 k}=2 \delta_{k 0}$

The orthonormal wavelet basis $\psi(x)$ associated with this multiresolution analysis is then defined by [17]

$\psi(x)=\sum_{n \in \mathbb{Z}} d_{n} \phi(2 x-n)$ 
where the wavelet coefficients

$d_{n}=(-1)^{n} C_{-n+1}$

With orthonormal wavelet bases for which the functions $\phi(x)$ and $\psi(x)$ are not compactly supported, infinitely many $C$ is different from zero. If $\phi(x)$ and $\psi(x)$ have compact support, then all but finitely many $C_{n}$ vanish, and the filters $h$ and $g$ have a finite number of "taps" (that is, nonzero entries $h_{n}, g_{n}$ ). For every orthonormal basis of compactly supported wavelets, there exists, therefore, an associated pair of finite filters for sub-band coding with exact reconstruction [18]. Exact reconstruction by orthogonal wavelets is only possible if

$\sum_{n} h_{n} h_{n+2 k}=\delta_{k 0}$

where $\delta_{I}$ is the usual delta sequence $\delta_{l, 0}=1$ if $I=0$ and 0 otherwise

$C_{n}=\sqrt{2} h_{n}$

$d_{n}=\sqrt{2} g_{n}$

where $h$ and $g$ are the finite impulse response (FIR) filters associated with $\phi(x)$ and $\psi(x)$. In [19], it is shown that for the orthogonal basis of wavelets the series $h_{n}$ and $g_{n}$, are such that,

$$
\begin{aligned}
& \sum_{n} h_{n}=\sqrt{2} \\
& \sum_{n} g_{n}=\sqrt{2}
\end{aligned}
$$

This condition makes it difficult to construct a Laguerre wavelet basis which is orthogonal. Therefore, we seek other means to tackle the problem. A possibility is through wavelet frames or biorthogonal wavelets. The idea here is to find a sequence that can generate Laguerre wavelets with the cascade algorithm, but at the same time, respect certain similarity conditions.

\subsection{The Construction of the Biorthogonal Laguerre wavelets basis}

The orthogonal wavelet construction schemes use the same filters for the decomposition and the reconstruction of a given signal. In the signal processing literature, there have been constructions of exact reconstruction filter banks whereby the decomposition filters are different from the reconstruction filters [7]. These filters permit more flexibility, are easier to design, and they have the advantage that symmetric filters can be obtained, which is not possible in the case where the decomposition and the reconstruction filters are identical. This property is very useful, given that Laguerre wavelets are symmetric.

Two dual bases $\psi_{m n}, \tilde{\psi}_{m n}$ are Biorthogonal wavelet bases if they are each given by the dilates and translates of one single function $\psi$ or $\tilde{\psi}$. Duality is defined as [7]

$\langle\tilde{\phi}, \phi(.-l)\rangle=\langle\tilde{\psi}, \psi(.-I)\rangle=\delta_{l, 0}$

In this scheme, we use four filters: $h$ and $g$ for the analysis while $\tilde{h}$ and $\tilde{g}$ are used for the synthesis of the signal. The scheme becomes like in Fig. 1

All four filters are related to their wavelets and scaling functions by the refinement equation. The relationships are as follows:

$\phi(x)=\sqrt{2} \sum_{n \in \mathbb{Z}} h_{n} \phi(2 x-n)$

$\psi(x)=\sqrt{2} \sum_{n \in \mathbb{Z}} g_{n} \phi(2 x-n)$

$\tilde{\phi}(x)=\sqrt{2} \sum_{n \in \mathbb{Z}} \tilde{h}_{n} \tilde{\phi}(2 x-n)$

$\tilde{\psi}(x)=\sqrt{2} \sum_{n \in \mathbb{Z}} \tilde{g}_{n} \tilde{\phi}(2 x-n)$

It is shown that the relationships between these filters are [19]:

$g_{n}=(-1)^{n+1} \tilde{h}_{-n}$

$\tilde{g}_{n}=(-1)^{n+1} h_{-n}$

The $z$ transform notation of these filters by the convention used here is:

$h(z)=\sum_{n \in \mathbb{Z}} h_{n} z^{n}$

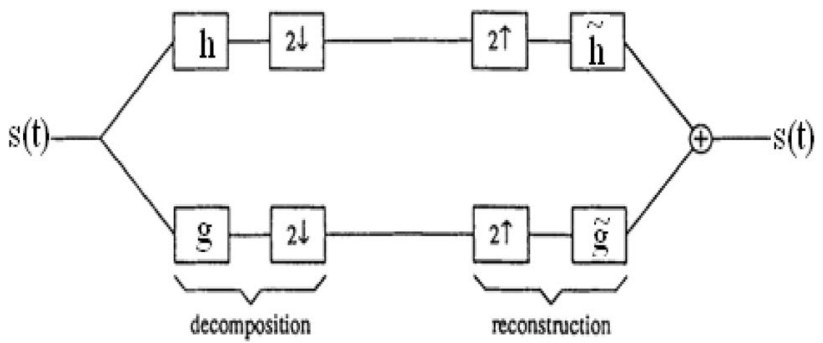

Fig. 1 Sub-band coding scheme with four different filters, two for decomposition and their duals for reconstruction [19] 
We consider only the case where the scaling function, the wavelet, and their duals are compactly supported. The consequence is that, we shall have a finite number of nonzero coefficients in the refinement Eqs. (15)-(18). In the field of signal processing, the set of filters are a set of finite biorthogonal filters if the following conditions are satisfied [7]:

$\frac{1}{2}[h(z) \overline{\tilde{h}}(z)+g(z) \overline{\tilde{g}}(z)]=1$

$\frac{1}{2}[h(-z) \overline{\tilde{h}}(z)-g(-z) \overline{\tilde{g}}(z)]=0$

The condition of (22) is often known as the perfect reconstruction condition while the condition of (23) is often known as the anti-aliasing condition. We usually refer to the four filters as biorthogonal quadruplets. Combining (23) and (22) yields

$h(z) \overline{\tilde{h}}(z)+h(-z) \stackrel{\overline{\tilde{g}}}{\bar{g}}(-z)=2$

In [7], the relationship between the low pass filter $h_{n}$ and its dual $\tilde{h}_{n}$ is given as:

$\sum_{n} h_{n} \tilde{h}_{n+2 k}=\delta_{k 0}$

We can notice that (25) is similar to (9) in the orthogonal case. (25) is very important in the construction because it suffices to obtain the filter coefficients $h_{n}$ and its dual can be got.

Let us suppose that the vector of the scaling filter (lowpass filter associated with the scaling function) has 8 coefficients, such that:

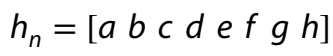

the wavelets are symmetric, so we render these coefficients symmetric. This yield

$h_{n}=\left[\begin{array}{llllllll}a & b & c & d & d & c & b & a\end{array}\right]$

In the same manner, the dual scaling filter (low pass filter associated with the dual scaling function) shall be

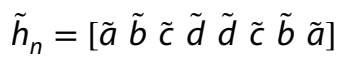

from (25) and for $\mathrm{k}=0$, we have:

$\sum_{n} h_{n} \tilde{h}_{n}=\delta_{00}=1$

Applying (29) to the filter coefficients yields

$2 \tilde{a} a+2 \tilde{b} b+2 \tilde{c} c+2 \tilde{d} d=1$

For $\mathrm{k}=1$, we have

$$
\begin{aligned}
& \sum_{n} h_{n} \tilde{h}_{n+2}=\delta_{10}=0 \\
& c \tilde{a}+d \tilde{b}+(a+d) \tilde{c}+(b+c) \tilde{d}=0
\end{aligned}
$$

For $\mathrm{k}=2$, we have

$\sum_{n} h_{n} \tilde{h}_{n+4}=\delta_{20}=0$

$d \tilde{a}+c \tilde{b}+b \tilde{c}+a \tilde{d}=0$

For $\mathrm{k}=3$, we have

$\sum_{n} h_{n} \tilde{h}_{n+6}=\delta_{30}=0$

$b \tilde{a}+a \tilde{b}=0$

We can see that we have a system of four equations and four unknowns, which give the matrices below:

$$
\begin{aligned}
& \left(\begin{array}{cccc}
2 a & 2 b & 2 c & 2 d \\
c & d & a+d & b+c \\
d & c & b & a \\
b & a & 0 & 0
\end{array}\right)\left[\begin{array}{l}
\tilde{a} \\
\tilde{b} \\
\tilde{c} \\
\tilde{d}
\end{array}\right]=\left[\begin{array}{l}
1 \\
0 \\
0 \\
0
\end{array}\right] \\
& \left(\begin{array}{cccc}
2 \tilde{a} & 2 \tilde{b} & 2 \tilde{c} & 2 \tilde{d} \\
\tilde{c} & \tilde{d} & \tilde{a}+\tilde{d} & \tilde{b}+\tilde{c} \\
\tilde{d} & \tilde{c} & \tilde{b} & \tilde{a} \\
\tilde{b} & \tilde{a} & 0 & 0
\end{array}\right)\left[\begin{array}{l}
a \\
b \\
c \\
d
\end{array}\right]=\left[\begin{array}{l}
1 \\
0 \\
0 \\
0
\end{array}\right]
\end{aligned}
$$

From (37) and (38), it is easily seen that the determination of the low pass filter coefficients can be obtained from the high pass filter coefficients and vice versa through the inversion of the respective $4 \times 4$ matrix.

\subsection{The proposed scheme}

The wavelets construction method we are proposing consists of several steps as can be seen in the scheme described in Fig. 2. Once an arbitrary sequence of symmetric low pass decomposition filters $h_{n}$ is determined, we use Eq. (25) to obtain the corresponding matrix. This matrix is inverted in order to obtain the other parameters that will generate the quadruplet of wavelets used in the cascade algorithm. We have selected this technique (Daubechies method of biorthogonal wavelets construction) because of the properties of the continuous Laguerre wavelets (symmetry, regularity and a family) which are similar to the properties of biorthogonal and reverse biorthogonal wavelets. In fact, biorthogonal wavelets in most of the cases are used as a starting point 


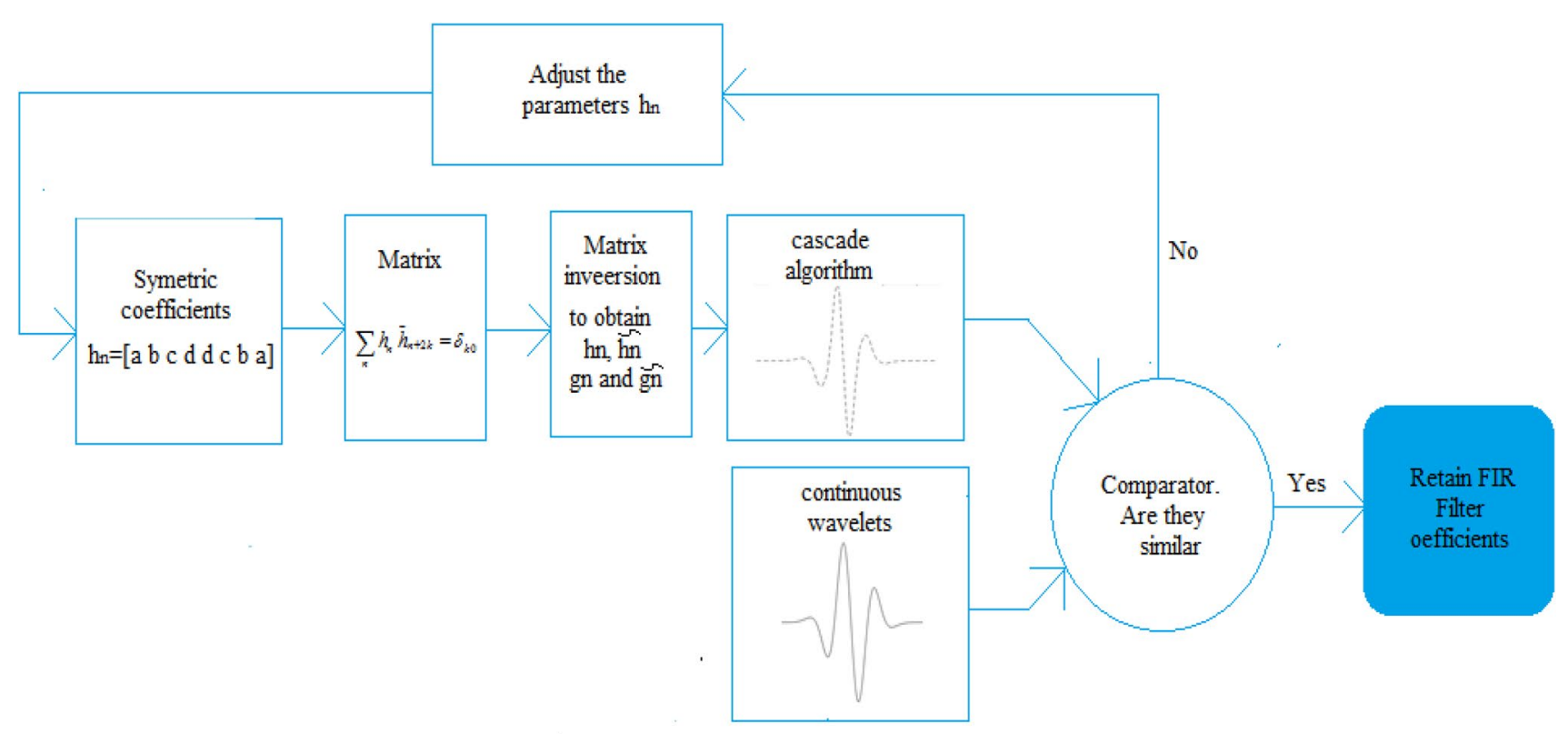

Fig. 2 The steps to obtain the Laguerre FIR filter coefficients from the continuous Laguerre wavelets

for the various iterations in order to determine the corresponding Laguerre wavelets.

The generated wavelets are compared with the required continuous wavelets using parameters like the mean squared error, maximum deviation and standard deviation. If both wavelets are similar enough, the corresponding FIR filter coefficients are retained, if not, the low pass decomposition filter parameters are adjusted and the process is repeated till the results are satisfactory. What makes our work novel is not only the objective, but the method as well. We have ameliorated the Daubechies method of biorthogonal wavelets construction by adding a stage of several iterations and comparing.

The cascade algorithm stage permits the determination of the wavelet function through its high pass filter counterpart over several iterations according to the mathematical law

$\psi(t)=\sum_{n=-\infty}^{\infty} g[n] \sqrt{2} \varphi^{(k)}(2 t-n)$

The corresponding Laguerre wavelets are given by Eqs. (1) and (2), meanwhile the comparator does a point by point comparing of the two functions in order to determine the mean squared error, maximum deviation and the standard deviation.

Where the statistical parameters are defined by [20] as follows:
$M S E r r=\frac{1}{N} \sum_{i=1}^{N}|s(i)-\hat{s}(i)|^{2}$

MADev $=\max _{i=1 \text { toN }}|s(i)-\hat{s}(i)|$

$S t D e v=\frac{1}{N} \sqrt{\sum_{i=1}^{N}(s(i)-\hat{s}(i))^{2}-\left[\sum_{i=1}^{N}(s(i)-\hat{s}(i))\right]^{2}}$

$s(i)$ is the continuous Laguerre wavelet, while $\hat{s}(i)$ is its discrete version generated by implementing the cascade algorithm and the number of samples is $\mathrm{N}$.

If the parameters are lower than a particular threshold, it means the two functions are very similar to each other, as such, the corresponding filter coefficients are retained. If not, the coefficients of the filters are adjusted and another iteration executed untill he required threshold is met.

We have used the graphical user interface of MATLAB R2016b software running on a 64 bits windows 10 operating system to write an algorithm that performs the operations step by step as described in Fig. 2. The example illustrated here is an 8-filter tab Laguerre 3 wavelet. The continuous wavelet (full line) is plot on the same axis with the cascade algorithm generated discrete wavelet (dotted line). We used 4 slide bars to adjust the parameters of the FIR filter coefficients continuously, while the results are plot instantaneously and compared (Fig. 3). 

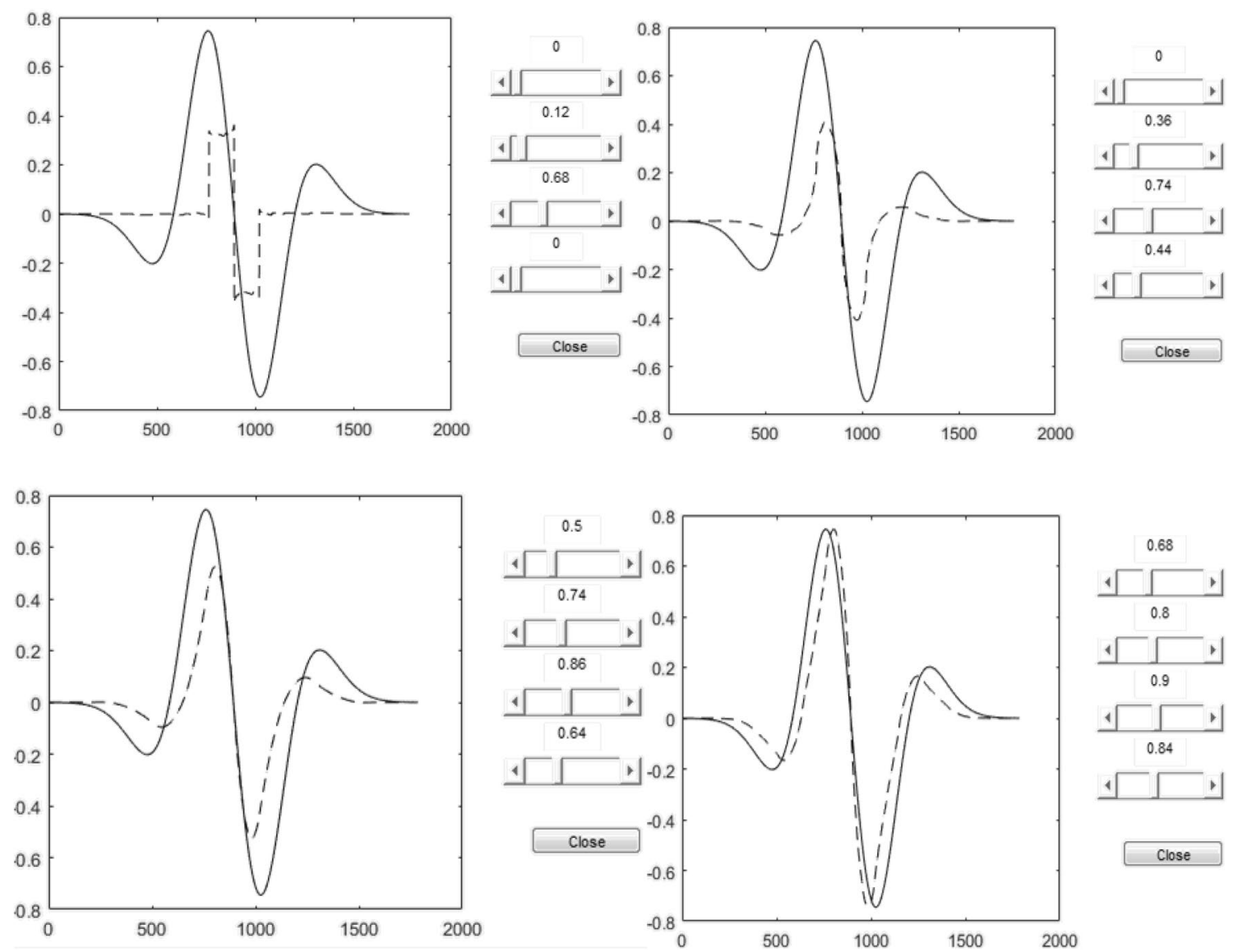

Fig. 3 Determining the FIR filter parameters that best suit an 8-filter tab Laguerre 3 wavelet by tuning the filter coefficients on the MATLAB GUI tool

The FIR filter coefficients that best suit our similarity requirements are retained.

From a more general perspective, this method can be used to obtain the FIR filter coefficients, and hence the DWT implementation of any continuous wavelet. In the process of pattern recognition in a signal for example, one can design continuous wavelets that look similar to a required pattern using the polynomial approximation or the ortho polynomial approximation method. However, such continuous wavelets can be implemented only using the CWT algorithm if their FIR filter coefficients are unknown. We hereby propose a method that can permit the tuning of some FIR filter coefficients that can be used for the DWT analysis in the pattern recognition of a signal, thereby saving much computation space and time.

This paper is not aimed at designing the best possible wavelets for signal analysis, but simply aimed at proposing a new wavelet tool which can be used just like many other wavelets with their strengths and weaknesses. In most signal processing tasks, the property of perfect reconstruction of a signal is the most important aspect of the scheme. In a signal compression task for example, once the signal can be perfectly reconstructed, it now suffices to apply a certain threshold to the wavelet coefficients in order to get some compression. This compression ratio depends on the said threshold, which affects the reconstructed signal as well.

We are proposing in this paper, a family of wavelets that have both analytical expression and FIR filter coefficients that can be used to perform several signal processing tasks as shown in Fig. 4.

\section{Presentation of results}

\subsection{The constructed discrete Laguerre wavelets}

The process of constructing the digital FIR filter coefficients took into consideration two main criteria, first of all, that the wavelet permits perfect reconstruction of a signal, and secondly, that the wavelet should resemble the corresponding continuous Laguerre wavelet as much as possible when generated with the cascade algorithm. As such, a vector of numbers was fine-tuned 


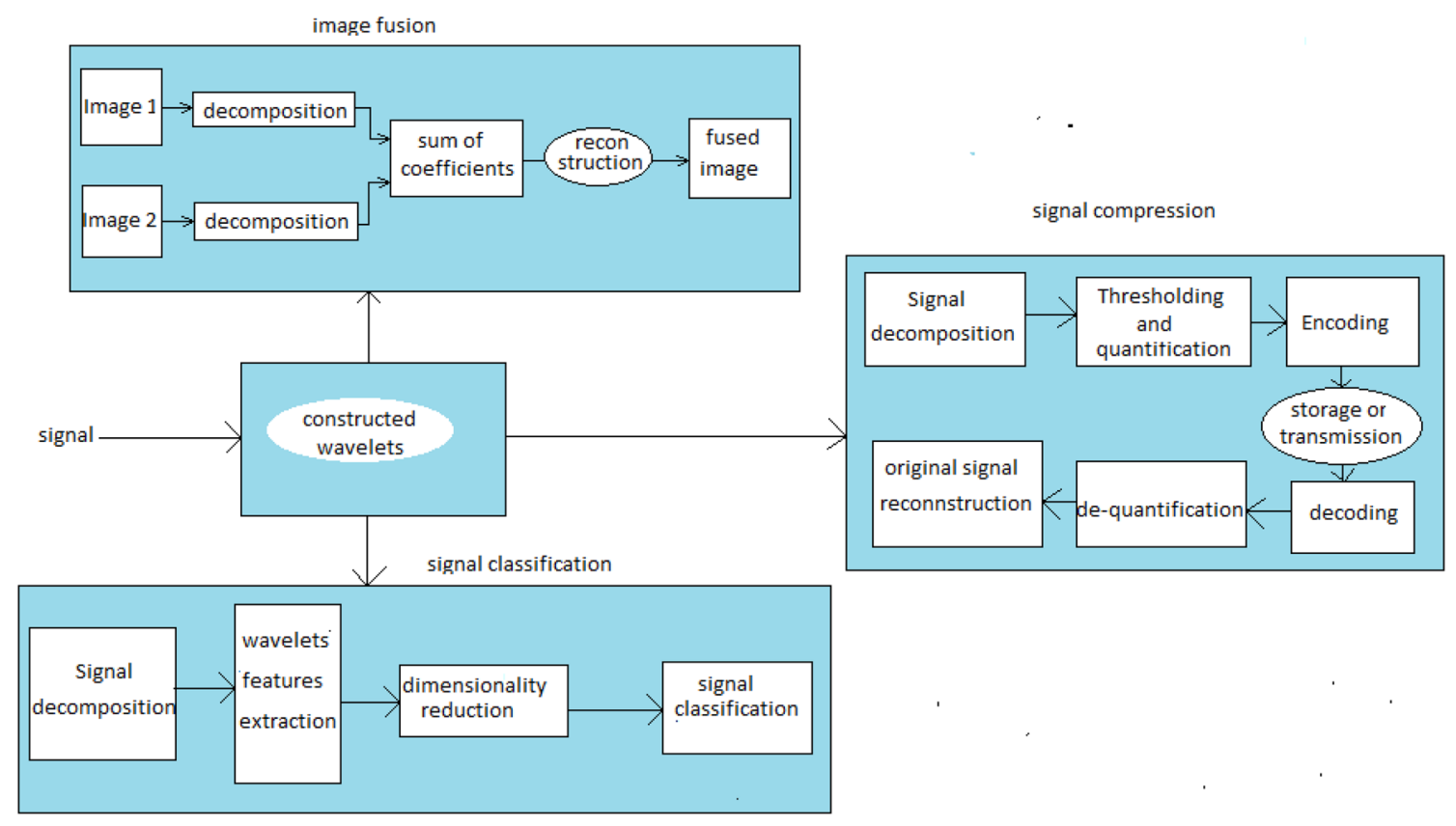

Fig. 4 The constructed wavelets can be used for signal compression, classification and fusion

using Eq. (25) and inverting the corresponding generated matrices to respect those two criteria. The results obtained in this work are coefficients of FIR filters that correspond to Laguerre wavelets. We have observed that, these filter coefficients are all different from all the other wavelets in the wavelets data base. In Table 1, we present the results obtained for the first fifteen members of the Laguerre wavelet family, alongside their quadruplet of FIR filter coefficients.

We can generate the corresponding wavelet by employing the cascade algorithm and compare their similarities with the continuous Laguerre wavelets. Once that is done, we can notice that the analysis wavelet corresponds to a shifted version of the Laguerre wavelets. Figures 5, 6 and 7 plots the Laguerre wavelets and the wavelet generated by these filter coefficients on the same scale.

For the Lag3 wavelet, we shall have to invert the following matrix corresponding to 12 FIR filter symmetric coefficients:

$\left(\begin{array}{cccccc}2 a & 2 b & 2 c & 2 d & 2 e & 2 f \\ c & d & a+e & b+f & c+f & d+e \\ e & f & f & e & a+d & b+c \\ f & e & d & c & b & a \\ d & c & b & a & 0 & 0 \\ b & a & 0 & 0 & 0 & 0\end{array}\right)\left[\begin{array}{c}\tilde{a} \\ \tilde{b} \\ \tilde{c} \\ \tilde{d} \\ \tilde{e} \\ \tilde{f}\end{array}\right]=\left[\begin{array}{l}1 \\ 0 \\ 0 \\ 0 \\ 0 \\ 0\end{array}\right]$

After performing the same operations as above, we obtain the following plots.
We can notice that the analysis wavelet corresponds to a shifted version of the Laguerre 3 and 4 wavelet respectively. Figure 7 plots the Laguerre 5 continuous wavelet and its discrete version obtained by the cascade algorithm.

\subsection{Discussion}

We have resorted to the Daubechies method of biorthogonal construction of wavelets to construct discrete Laguerre wavelets. The biorthogonal wavelets are not orthogonal, but not having to be orthogonal gives more options to a variety of filters such as symmetric filters thus allowing them to possess the symmetric property. The Biorthogonal analysis is possible with perfect reconstruction if a pair of filters are used for the analysis and another for the synthesis as shown above in Table 1.

When applied to model a signal, the reconstruction is perfect, using these wavelets. The Laguerre discrete wavelets are constructed to resemble the continuous version as much as possible, and at the same time, permit the perfect reconstruction of a digital signal. We used objective parameters like the mean squared error, the maximum deviation, and the standard deviation to quantify the differences between the continuous and the discrete wavelets obtained from the cascade algorithm. It is seen from Figs. 5, 6 and 7 that, these parameters are close to zero, meaning that the two wavelets are very similar to each other. These wavelets can serve in several applications like data compression, denoising, filtering, etc. 
Table 1 The first fifteen members of the Laguerre wavelet family FIR filter coefficients

\begin{tabular}{|c|c|}
\hline Wavelets & FIR filter coefficients \\
\hline \multicolumn{2}{|l|}{ Laguerre 1} \\
\hline$h_{n}$ & 0.01270 .04920 .16310 .22590 .22590 .16310 .04920 .0127 \\
\hline$\tilde{h}_{n}$ & $-1.37695 .3247-4.283911 .445011 .4450-14.28395 .3247-1.3769$ \\
\hline$g_{n}$ & $1.37695 .324714 .283911 .4450-11.4450-14.2839-5.3247-1.3769$ \\
\hline$\tilde{g}_{n}$ & $0.0127-0.04920 .1631-0.22590 .2259-0.16310 .0492-0.0127$ \\
\hline \multicolumn{2}{|l|}{ Laguerre 2} \\
\hline$h_{n}$ & $00-0.0564-0.03550 .37600 .71040 .3760-0.0355-0.05640$ \\
\hline$\tilde{h}_{n}$ & $0.0378-0.0238-0.11060 .38740 .98270 .3874-0.1106-0.02380 .03780$ \\
\hline$g_{n}$ & $-0.0378-0.02380 .11060 .3874-0.98270 .38740 .1106-0.0238-0.03780$ \\
\hline$\tilde{g}_{n}$ & $00-0.05640 .03550 .3760-0.71040 .37600 .0355-0.05640$ \\
\hline \multicolumn{2}{|l|}{ Laguerre 3} \\
\hline$h_{n}$ & $00-0.0014-0.00440 .16810 .51610 .51610 .1681-0.0044-0.001400$ \\
\hline$\tilde{h}_{n}$ & $-0.01380 .04140 .0525-0.2679-0.06180 .98670 .9867-0.0618-0.26790 .05250 .0414-0.0138$ \\
\hline$g_{n}$ & $0.01380 .0414-0.0525-0.26790 .06180 .9867-0.9867-0.06180 .26790 .0525-0.0414-0.0138$ \\
\hline$\tilde{g}_{n}$ & $00-0.00140 .00440 .1681-0.51610 .5161-0.1681-0.00440 .001400$ \\
\hline \multicolumn{2}{|l|}{ Laguerre 4} \\
\hline$h_{n}$ & $\begin{array}{l}00-0.0002-0.00020 .01450 .0141-0.0766-0.04420 .40240 .73690 .4024-0.0442 \\
-0.07660 .01410 .0145-0.0002-0.00020\end{array}$ \\
\hline$\tilde{h}_{n}$ & $\begin{array}{l}0.0019-0.0019-0.01700 .01190 .0497-0.0773-0.09410 .44080 .84590 .4408-0.0941 \\
-0.07730 .04970 .0119-0.0170-0.00190 .00190\end{array}$ \\
\hline$g_{n}$ & $\begin{array}{l}-0.0019-0.00190 .01700 .0119-0.0497-0.07730 .09410 .4408-0.84590 .44080 .0941 \\
-0.0773-0.04970 .01190 .0170-0.0019-0.00190\end{array}$ \\
\hline$\tilde{g}_{n}$ & $\begin{array}{l}00-0.00020 .00020 .0145-0.0141-0.07660 .04420 .4024-0.73690 .40240 .0442 \\
-0.0766-0.01410 .01450 .0002-0.00020\end{array}$ \\
\hline \multicolumn{2}{|l|}{ Laguerre 5} \\
\hline$h_{n}$ & $0.00380 .01140 .0118-0.01410 .20110 .48320 .48320 .2011-0.01410 .01180 .01140 .0038$ \\
\hline$\tilde{h}_{n}$ & $-0.01380 .04140 .0525-0.3379-0.08181 .05671 .0567-0.0818-0.33790 .05250 .0414-0.0138$ \\
\hline$g_{n}$ & $0.01380 .0414-0.0525-0.33790 .08181 .0567-.0567-0.08180 .33790 .0525-0.0414-0.0138$ \\
\hline$\tilde{g}_{n}$ & $0.0038-0.01140 .01180 .01410 .2011-0.48320 .4832-0.2011-0.0141-0.01180 .0114-0.0038$ \\
\hline \multicolumn{2}{|r|}{ ( } \\
\hline$h_{n}$ & $\begin{array}{l}00-0.0002-0.00020 .01550 .0151-0.0806-0.04620 .41550 .77700 .4155 \\
-0.0462-0.08060 .01510 .0155-0.0002-0.00020\end{array}$ \\
\hline \multirow{2}{*}{$\tilde{h}_{n}^{\tilde{h}}$} & $0.0019-0.0019-0.01700 .01190 .0497-0.0773-0.09410 .44080 .78590 .4408$ \\
\hline & $-0.0941-0.07730 .04970 .0119-0.0170-0.00190 .00190$ \\
\hline$g_{n}$ & $\begin{array}{l}-0.0019-0.00190 .01700 .0119-0.0497-0.07730 .09410 .4408-0.78590 .4408 \\
0.0941-0.0773-0.04970 .01190 .0170-0.0019-0.0019\end{array}$ \\
\hline$\tilde{g}_{n}$ & $\begin{array}{l}00-0.00020 .00020 .0155-0.0151-0.08060 .04620 .4155-0.77700 .4155 \\
0.0462-0.0806-0.01510 .01550 .0002-0.00020\end{array}$ \\
\hline \multicolumn{2}{|l|}{ Laguerre 7} \\
\hline \multirow[t]{2}{*}{$h_{n}$} & 0.00020 .00060 .00270 .00600 .01990 .03670 .17290 .43020 .43020 .1729 \\
\hline & 0.03670 .01990 .00600 .00270 .00060 .0002 \\
\hline$\tilde{h}_{n}$ & $\begin{array}{l}0.0030-0.0091-0.01680 .08470 .0113-0.3512-0.27651 .30161 .3016-0.2765 \\
-0.35120 .01130 .0847-0.0168-0.00910 .0030\end{array}$ \\
\hline$g_{n}$ & $\begin{array}{l}-0.0030-0.00910 .01680 .0847-0.0113-0.35120 .27651 .3016-1.3016-0.2765 \\
0.35120 .0113-0.0847-0.01680 .00910 .0030\end{array}$ \\
\hline$\tilde{g}_{n}$ & $\begin{array}{l}0.0002-0.00060 .0027-0.00600 .0199-0.03670 .1729-0.43020 .4302-0.1729 \\
0.0367-0.01990 .0060-0.00270 .0006-0.0002\end{array}$ \\
\hline Laguerre 8 & \\
\hline
\end{tabular}


Table 1 (continued)

\begin{tabular}{|c|c|}
\hline Wavelets & FIR filter coefficients \\
\hline$h_{n}$ & $\begin{array}{l}00000.01850 .0185-0.1009-0.05180 .53570 .97300 .5357-0.0518 \\
-0.10090 .01850 .0185000\end{array}$ \\
\hline$\tilde{h}_{n}$ & $\begin{array}{l}0.0015-0.0015-0.01330 .00930 .0388-0.0603-0.07340 .32820 .6442 \\
0.3282-0.0734-0.06030 .03880 .0093-0.0133-0.00150 .00150\end{array}$ \\
\hline$g_{n}$ & $\begin{array}{l}-0.0015-0.00150 .01330 .0093-0.0388-0.06030 .07340 .3282-0.6442 \\
0.32820 .0734-0.0603-0.03880 .00930 .0133-0.0015-0.00150\end{array}$ \\
\hline$\tilde{g}_{n}$ & $\begin{array}{l}00000.0185-0.0185-0.10090 .05180 .5357-0.97300 .53570 .0518 \\
-0.1009-0.01850 .0185000\end{array}$ \\
\hline \multicolumn{2}{|l|}{ Laguerre 9} \\
\hline$h_{n}$ & $\begin{array}{l}0.00040 .00120 .00150 .00120 .01250 .04180 .15160 .45020 .45020 .1516 \\
0.04180 .01250 .00120 .00150 .00120 .0004\end{array}$ \\
\hline$\tilde{h}_{n}$ & $\begin{array}{l}0.0030-0.0091-0.01680 .07470 .0313-0.3512-0.17651 .20161 .2016 \\
-0.1765-0.35120 .03130 .0747-0.0168-0.00910 .0030\end{array}$ \\
\hline$g_{n}$ & $\begin{array}{l}-0.0030-0.00910 .01680 .0747-0.0313-0.35120 .17651 .2016-1.2016 \\
-0.17650 .35120 .0313-0.0747-0.01680 .00910 .0030\end{array}$ \\
\hline$\tilde{g}_{n}$ & $\begin{array}{l}0.0004-0.00120 .0015-0.00120 .0125-0.04180 .1516-0.45020 .45020 .1516 \\
-0.04180 .0125-0.00120 .0015-0.00120 .0004\end{array}$ \\
\hline Laguerre 10 & \\
\hline$h_{n}$ & $\begin{array}{l}00000.01660 .0166-0.0905-0.04640 .48030 .87230 .4803 \\
-0.0464-0.09050 .01660 .0166000\end{array}$ \\
\hline$\tilde{h}_{n}$ & $\begin{array}{l}0.0017-0.0017-0.01480 .01040 .0433-0.0672-0.08180 .36610 .7186 \\
0.3661-0.0818-0.06720 .04330 .0104-0.0148-0.00170 .00170\end{array}$ \\
\hline$g_{n}$ & $\begin{array}{l}-0.0017-0.00170 .01480 .0104-0.0433-0.06720 .08180 .3661-0.7186 \\
0.36610 .0818-0.0672-0.04330 .01040 .0148-0.0017-0.00170\end{array}$ \\
\hline$\tilde{g}_{n}$ & $\begin{array}{l}00000.0166-0.0166-0.09050 .04640 .4803-0.87230 .4803 \\
0.0464-0.0905-0.01660 .0166000\end{array}$ \\
\hline Laguerre 11 & \\
\hline$h_{n}$ & $\begin{array}{l}0.00080 .00250 .0017-0.00140 .01320 .06830 .13250 .47110 .47110 .1325 \\
0.06830 .0132-0.00140 .00170 .00250 .0008\end{array}$ \\
\hline$\tilde{h}_{n}$ & $\begin{array}{l}0.0030-0.0091-0.01680 .07470 .0303-0.3812-0.12651 .15161 .1516 \\
-0.1265-0.38120 .03030 .0747-0.0168-0.00910 .0030\end{array}$ \\
\hline$g_{n}$ & $\begin{array}{l}-0.0030-0.00910 .01680 .0747-0.0303-0.38120 .12651 .1516-1.1516 \\
-0.12650 .38120 .0303-0.0747-0.01680 .00910 .0030\end{array}$ \\
\hline$\tilde{g}_{n}$ & $\begin{array}{l}0.0008-0.00250 .00170 .00140 .0132-0.06830 .1325-0.47110 .4711-0.1325 \\
0.0683-0.0132-0.0014-0.00170 .0025-0.0008\end{array}$ \\
\hline Laguerre 12 & \\
\hline$h_{n}$ & $\begin{array}{l}00000.01440 .0145-0.0787-0.04040 .41780 .75890 .4178-0.0404 \\
-0.07870 .01450 .0144000\end{array}$ \\
\hline$\tilde{h}_{n}$ & $\begin{array}{l}0.0019-0.0019-0.01700 .01190 .0497-0.0773-0.09410 .42080 .8259 \\
0.4208-0.0941-0.07730 .04970 .0119-0.0170-0.00190 .00190\end{array}$ \\
\hline$g_{n}$ & $\begin{array}{l}-0.0019-0.00190 .01700 .0119-0.0497-0.07730 .09410 .4208-0.8259 \\
0.42080 .0941-0.0773-0.04970 .01190 .0170-0.0019-0.00190\end{array}$ \\
\hline$\tilde{g}_{n}$ & $\begin{array}{l}00000.0144-0.0145-0.07870 .04040 .4178-0.75890 .41780 .0404 \\
-0.0787-0.01450 .0144000\end{array}$ \\
\hline Laguerre 13 & \\
\hline$h_{n}$ & $\begin{array}{l}0.00010 .00040 .0001-0.00080 .00310 .01160 .02320 .03790 .24740 .5006 \\
0.50060 .24740 .03790 .02320 .01160 .0031-0.00080 .00010 .00040 .0001\end{array}$ \\
\hline$\tilde{h}_{n}$ & $\begin{array}{l}-0.00070 .00200 .0051-0.0206-0.01410 .09910 .0123-0.3202-0.34791 .1921 \\
1.1921-0.3479-0.32020 .01230 .0991-0.0141-0.02060 .00510 .0020-0.0007\end{array}$ \\
\hline
\end{tabular}


Table 1 (continued)

\begin{tabular}{|c|c|}
\hline Wavelets & FIR filter coefficients \\
\hline$g_{n}$ & $\begin{array}{l}0.00070 .0020-0.0051-0.02060 .01410 .0991-0.0123-0.32020 .34791 .1921 \\
-1.1921-0.34790 .32020 .0123-0.0991-0.01410 .02060 .0051-0.00200 .0007\end{array}$ \\
\hline$\tilde{g}_{n}$ & $\begin{array}{l}0.0001-0.00040 .00010 .00080 .0031-0.01160 .0232-0.03790 .2474-0.5006 \\
0.5006-0.24740 .0379-0.02320 .0116-0.0031-0.0008-0.00010 .0004-0.0001\end{array}$ \\
\hline Laguerre 1 & \\
\hline$h_{n}$ & $\begin{array}{l}00-0.0001-0.00010 .01570 .0155-0.0824-0.04450 .42610 .7962 \\
0.4261-0.0445-0.08240 .01550 .0157-0.0001-0.00010\end{array}$ \\
\hline$\tilde{h}_{n}$ & $\begin{array}{l}0.0019-0.0019-0.01700 .01190 .0497-0.0773-0.09410 .44080 .84590 .4408-0.0941 \\
-0.07730 .04970 .0119-0.0170-0.00190 .00190\end{array}$ \\
\hline$g_{n}$ & $\begin{array}{l}-0.0019-0.00190 .01700 .0119-0.0497-0.07730 .09410 .4308-0.7659 \\
0.43080 .0941-0.0773-0.04970 .01190 .0170-0.0019-0.00190\end{array}$ \\
\hline$\tilde{g}_{n}$ & $\begin{array}{l}00-0.00010 .00010 .0157-0.0155-0.08240 .04450 .4261-0.7962 \\
0.42610 .0445-0.0824-0.01550 .01570 .0001-0.00010\end{array}$ \\
\hline Laguerre 15 & \\
\hline$h_{n}$ & $\begin{array}{l}00.00010 .00010 .00000 .00170 .00510 .01710 .03750 .19170 .4439 \\
0.44390 .19170 .03750 .01710 .00510 .001700 .00010 .00010\end{array}$ \\
\hline$\tilde{h}_{n}$ & $\begin{array}{l}-0.00070 .00200 .0051-0.0206-0.01410 .09910 .0123-0.3202-0.34791 .3021 \\
1.3021-0.3479-0.32020 .01230 .0991-0.0141-0.02060 .00510 .0020-0.0007\end{array}$ \\
\hline$g_{n}$ & $\begin{array}{l}0.00070 .0020-0.0051-0.02060 .01410 .0991-0.0123-0.32020 .34791 .3021 \\
-1.3021-0.34790 .32020 .0123-0.0991-0.01410 .02060 .0051-0.0020-0.0007\end{array}$ \\
\hline$\tilde{g}_{n}$ & $\begin{array}{l}0-0.00010 .0001-0.00000 .0017-0.00510 .0171-0.03750 .1917-0.4439 \\
0.4439-0.19170 .0375-0.01710 .0051-0.001700 .0001-0.00010\end{array}$ \\
\hline
\end{tabular}
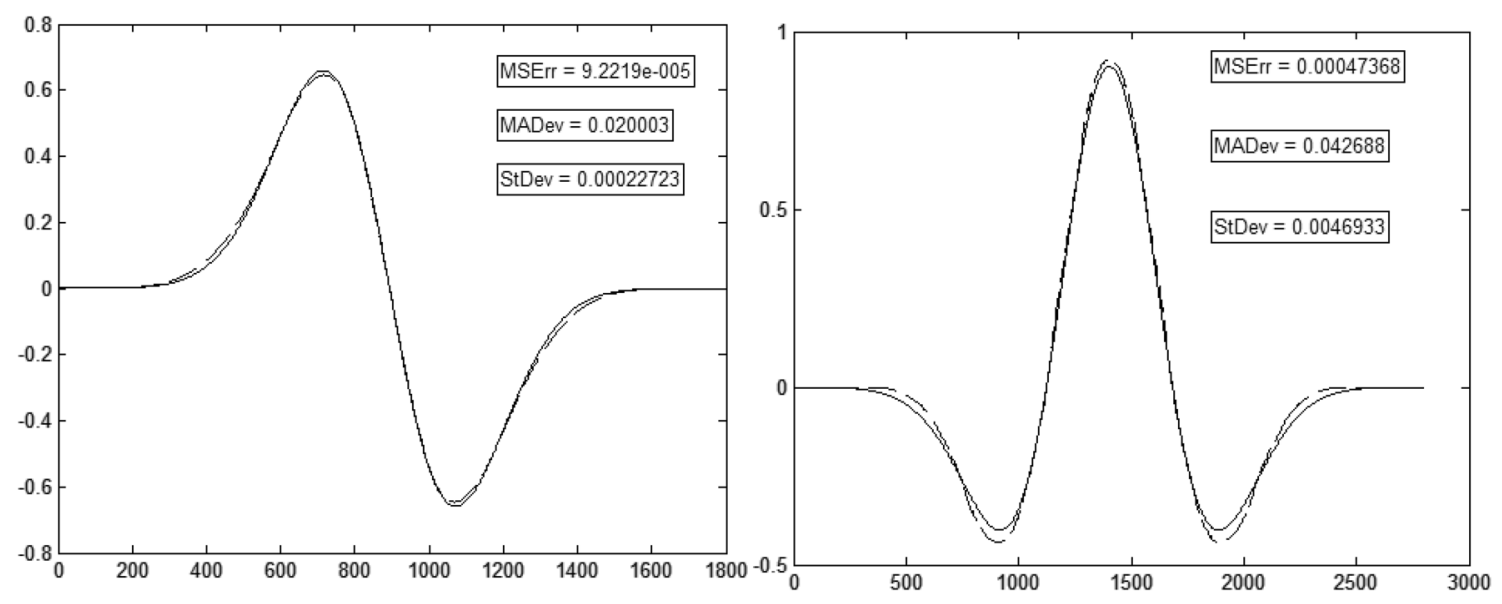

Fig. 5 A plot of the Laguerre 1 and 2 wavelets ----, and their discrete versions

generated by the cascade algorithm

We have used the first five members of the discrete Laguerre wavelet family to perform the same analysis as in [5]. Here, the wavelets are used in association with the artificial neural network and support vector machines in order to classify EEG (Electro encephalograph) epileptic signals as seizure or none seizure. The idea was to compare the performance of the discrete Laguerre wavelets implemented with the DWT to the continuous Laguerre wavelets counterpart, implemented with the CWT. The method consisted of decomposing each signal up to level 8 and for each level we computed some features to serve as feature vectors for the classifiers. These features included the mean, minimum, maximum, standard deviation, percentage power, Shannon and wavelet entropy of the approximation and detail coefficients. The results of this experiment were not very different from that conducted with the CWT in terms of classification accuracy (for each classification case, the CA was on average $\pm 2 \%$ compared 

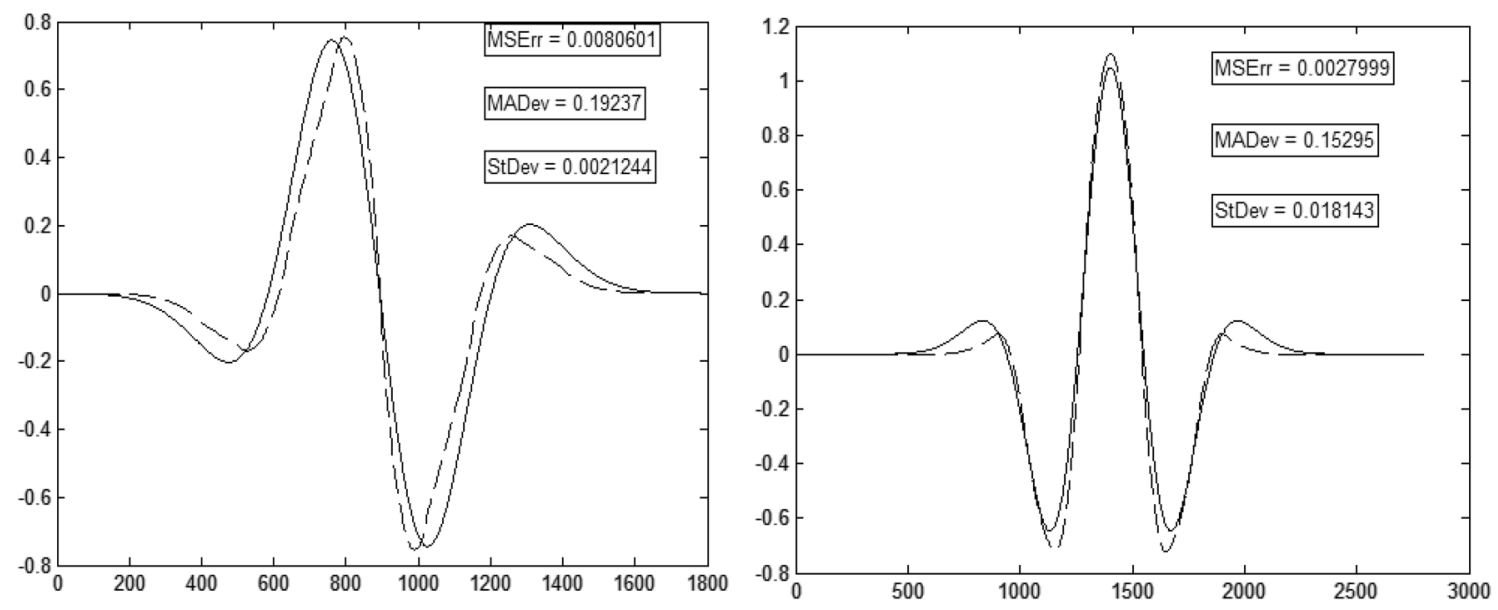

Fig. 6 Plot of the Laguerre 3 and 4 continuous wavelets , and their discrete version generated by the cascade algorithm ----

to the CWT experiment). However, as we expected, the computation time was greatly reduced. This is due to the fact that, the DWT is faster than the CWT. It took on average only about $20 \%$ of the CWT computation time to perform the same classification task with DWT.

\section{Conclusion}

The goal in this paper is to propose a new wavelet tool which can be used for signal analysis. We started by determining the Finite impulse response filter coefficients that correspond to the Laguerre wavelets which will permit a fast wavelet transform analysis implementation. This was done by the help of biorthogonal wavelets, which respect the constraints of perfect reconstruction

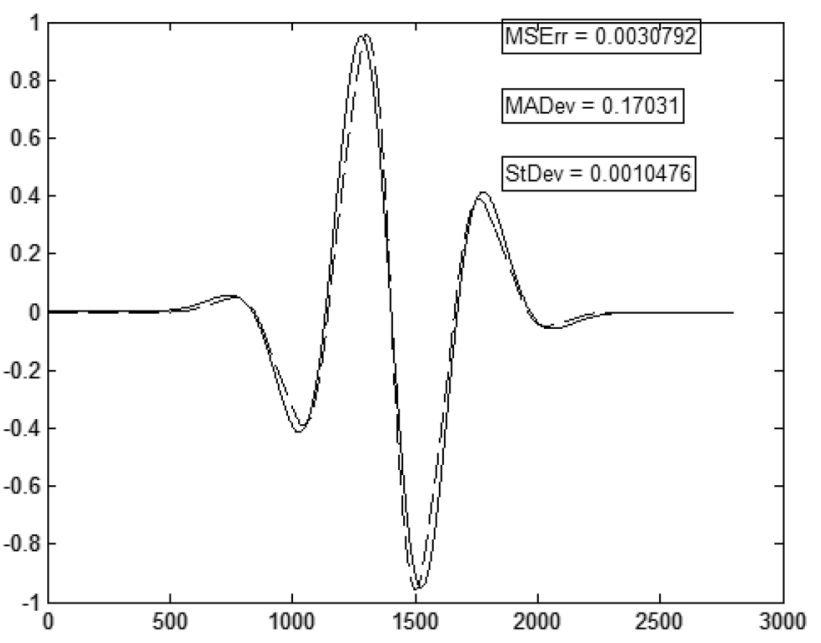

Fig. 7 Plot of the Laguerre 5 wavelet and its discrete version generated by the cascade algorithm --- and anti-aliasing, and at the same time look as close to the continuous wavelets as possible. Contrary to continuous wavelets like the Mexican hat wavelet which has an explicit expression and no FIR filters, or discrete wavelets like Daubechies wavelets that have no analytical expression but FIR filter coefficients, the proposed Laguerre wavelets have both analytical expressions and FIR filter coefficients. This makes them suitable to be used both in the discrete wavelet transform and the continuous wavelet transform algorithms as well. This research work will permit in the future the determination of the discrete versions of several continuous wavelets. In the wavelets data base, most wavelets are either continuous (with a mathematical expression but no FIR filter coefficients) or discrete (with a FIR filter coefficient but no mathematical expression). The specialty of the Laguerre wavelets is that, they have both a continuous and a discrete version. The fast wavelet transform algorithm can also be used in several signal analysis applications with these filter coefficients such as signal de-noising, compression, image fusion, just to name a few. In the future, we shall work on the applications of the constructed Laguerre wavelet filters in several biomedical signal processing tasks like the brain control interface $(\mathrm{BCl})$, and some digital signal processing tasks like image fusion, filtering, and compression.

\section{Compliance with ethical standards}

Conflict of interest On behalf of all authors, the corresponding author states that there is no conflict of interest. 


\section{References}

1. Workman MJ, Serov A, Halevi B, Atanassov P, Artyushkova K (2015) Application of the discrete wavelet transform to SEM and AFM micrographs for quantitative analysis of complex surfaces. Langmuir 31(17):4924-4933. https://doi.org/10.1021/acs.langm uir.5b00292

2. Sharma R, Pachori RB, Acharya UR (2015) An integrated index for the identification of focal electroencephalogram signals using discrete wavelet transform and entropy measures. Entropy 17(8):5218-5240. https://doi.org/10.3390/e17085218

3. Tchiotsop D (2007) Modelisations polynomiales des signaux ECG. Application a la compression. Ph.D. thesis in physics. Institut National Polytechnique de Lorraine

4. Bruno Peachap A, Tchiotsop D (2018) Some new continuous wavelets based on laguerre polynomials applied in pattern detection of noisy signals. Int J Adv Res Electr Electron Instrum Eng 7(2):2-7

5. BrunoPeachap A, Tchiotsop D (2019) Epileptic seizures detection based on some new Laguerre polynomial wavelets, artificial neural networks and support vector machines. Inform Med Unlocked. https://doi.org/10.1016/j.imu.2019.100209

6. Sarkar TK, Su C, Adve R, Salazar-Palma M, Garcia-Castillo L, Boix RR (1998) A tutorial on wavelets from an electrical engineering perspective. I. Discrete wavelet techniques. IEEE Antennas Propag. Mag. 40(5):49-68

7. Cohen A, Daubechies I, Feauveau J (1992) Bi-orthogonal bases of compactly supported wavelets. Commun Pure Appl Math 45:485-560

8. Sweldens W (1995) The lifting scheme: a custom-design construction of biorthogonal wavelets. AT\&T Bell Laboratories, Murray Hill

9. Haar A (1910) ZurTheorie der orthogonalen Funktionensysteme. Math Ann 69(3):331-371. https://doi.org/10.1007/BF01456326

10. Mallat $S$ (2009) A wavelet tour of signal processing: the sparse way. 3rd edn. Academic Press, Cambridge, 1998
11. Nielsen M (1999) On the construction and frequency localization of finite orthogonal quadrature filters. J Approx Theory 108:36-52

12. Zhang L, Arain MB, Bhatti MM, Zeeshan A, Hal-Sulami H (2020) Effects of magnetic Reynolds number on swimming of gyrotactic microorganisms between rotating circular plates filled with nanofluids. Appl Math Mech 41(4):637-654. https://doi. org/10.1007/s10483-020-2599-7

13. Muhammad B, As As, Tehseen A, Sultan A, Rahmat E (2020) Study of activation energy on the movement of gyrotactic microorganism in a magnetized nanofluids past a porous plate. Processes 8(3):328

14. Tuma M (2000) Application of Laguerre functions to data compression. GRANT J 343:1-5

15. Koekoek R (1987) Generalizations of Laguerre (type) polynomials. PhD Thesis, Delft University of Technology, Faculty of Mathematics and Informatics

16. Misiti M, Misiti Y, Oppenheim G, Poggi JM (2007) Wavelets and their applications. Printed and bound in Great Britain by Antony Rowe Ltd, Chippenham, Wiltshire. ISTE Ltd, pp 118-133

17. Polikar R (1991) The story of wavelets. SP Mag 10:2-4

18. Liu C-L (2010) A tutorial of the wavelet transform, vol 331. Prentice-Hall, Upper Saddle River, pp 6-20

19. Daubechies I (1992) Ten lectures on wavelets. In: CBMS-NSF regional conference series in applied mathematics, vol. 61. Society for Industrial and Applied Mathematics (SIAM), Philadelphia

20. Tchiotsop D, Dorr VL, Talla PK, Fogué M (2015) Compression and analysis of ECG feature using optimal polynomial approximations. Int J Eng Res Manag 2(7):107-114

Publisher's Note Springer Nature remains neutral with regard to jurisdictional claims in published maps and institutional affiliations. 